\title{
Evaluation of mortality and acute kidney injury by KDIGO and RIFLE in patients treated with colistin in the intensive care unit
}

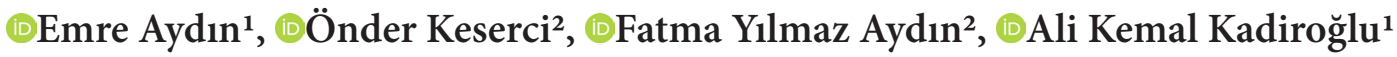 \\ ${ }^{1}$ University of Dicle, Faculty of Medicine, Department of Nephrology, Diyarbakır, Turkey \\ ${ }^{2}$ University of Dicle, Faculty of Medicine, Department of Internal Medicine, Diyarbakır, Turkey
}

Cite this article as: Aydın E, Keserci Ö, Yılmaz Aydın F, Kadiroğlu AK. Evaluation of mortality and acute kidney injury by KDIGO and RIFLE in patients treated with colistin in the intensive care unit. J Health Sci Med 2021; 4(5): 610-614.

\begin{abstract}
Introduction: Multidrug-resistant bacterial infections such as pseudomonas aeruginosa, Acinetobacter baumannii, klebsiella pneumoniae are common in Intensive Care Units. Colistin is preferred today due to limited treatment options sensitive to these pathogens. However, colistin has a high potential for nephrotoxicity. Some classifications (KDIGO, RIFLE, AKIN) are used for the diagnosis of Acute Kidney Injury (AKI). The incidence of AKI varies according to these classifications. In our study, we aimed to evaluate and compare the development of Acute Kidney Injury according to KDIGO criteria and RIFLE classification in patients followed up in the intensive care unit and started colistin therapy.

Materials and Method: The data of 145 patients who started colistin treatment in the Internal Medicine Intensive Care Unit between January 2016 and December 2019 were retrospectively reviewed. Patients were grouped as those with Acute Kidney Injury and those without. Acute Kidney Injury evaluation was made according to RIFLE classification and KDIGO criteria.

Results: In patients who received colistin treatment, 75 patients were evaluated as AKI when KDIGO criteria were used for the AKI diagnosis, and 54 patients were evaluated as AKI when the RIFLE classification was used. While the number of KDIGO Stage 1 patients was 44, the number of patients in the RIFLE classification risk group was determined as 23 . The mean SOFA score was calculated as 7.91 \pm 2.8 and the mean APACHE II score as $22.77 \pm 5.3$. The duration of stay in the ICU was $22.60 \pm 9.04$. The duration of stay in the ICU was shorter in the group who developed AKI. $44.8 \%$ of the patients included in the study died. $52 \%$ of patients who developed AKI and $37.1 \%$ of those who did not develop AKI died. 30-day mortality in patients with AKI was significantly higher than both KDIGO staging and RIFLE classification $(p=0.03, p=0.005$, respectively). Mortality rate increased 1.42 times in KDIGO Stage 1, 2.79 times in KDIGO Stage 2 and 2.10 times in KDIGO Stage 3. When evaluated by the RIFLE classification, the mortality rate increased 2.32 times in the Risk group, 3.12 times in the Injury group, 2.06 times in the Failure group and 2.15 times in the Loss group.

Conclusion: We observed an increase in the frequency of AKI in patients using colistin in the ICU, both according to KDIGO criteria and the RIFLE classification. This situation also increases the 30 -day mortality. We think that the KDIGO criteria are more sensitive to diagnose AKI in these patients.
\end{abstract}

Keywords: Colistin, acute kidney injury, intensive care units

\section{INTRODUCTION}

In recent years, an increase in multi-drug resistant (MDR) bacterial infections has been observed in patients followed in Intensive Care Units (ICU). These infections prolong hospital stay and increase mortality and morbidity (1). Colistin is preferred among polymyxin group polypeptide antibiotics, due to thelimited treatment options available that are sensitive to these multidrugresistant pathogens such as Acinetobacter baumannii, pseudomonas aeruginosa, klebsiella pneumoniae (2).

Polymyxins are divided into 5 groups (A-E) and some of them (A, C, D) are not used today due to their severe toxicity. Only polymyxin $\mathrm{B}$ and polymyxin $\mathrm{E}$ (also known as colistin) are in clinical use (3). The most important side effect of colistin is nephrotoxicity. As a result of the studies conducted, the rate of developing acute kidney injury (AKI) after colistin treatment ranges between 20-60\% (4). Colistin causes cell swelling and cell lysis by increasing the permeability of the tubular epithelial 
cell membrane. Nephrotoxicity is dose dependent and reversible, although not always (5). Early recognition of nephrotoxicity can be achieved with closer follow-up in patients who were given colistin. RIFLE (Risk, Injury, Failure, Loss, End stage kidney disease) classification for AKI definition and Kidney Disease Improving Global Outcomes (KDIGO) criteria are used. In our study, we aimed to determine the development of AKI in patients receiving colistin treatment in intensive care units as per the RIFLE classification and KDIGO criteria, and its correlation with the risk factors related to nephrotoxicity, frequency of nephrotoxicity and mortality.

\section{MATERIAL AND METHOD}

Approval for the study was granted by the Ethics Committee of Dicle University Medical Faculty (Date: 05.03.2020, Decision No: 254). All procedures were carried out in accordance with the ethical rules and the principles of the Declaration of Helsinki.

The data of 145 patients who received colistin treatment in the General Internal Medicine Intensive Care Unit between 2016 and 2019 were retrospectively analyzed. Patients $>18$ years of age who received colistin treatment for longer than 48 hours, regardless of gender, were included in the study. Patients with pre-treatment acute kidney injury and chronic kidney damage who received colistin for less than 48 hours were excluded from the study. Colistin was administered to the patients at a dose of 300 $\mathrm{mg} /$ day. Demographic characteristics (age and gender) of the patients were evaluated. Acute physiology and chronic health evaluation II (APACHE II) and sequential organ failure assessment (SOFA) score were calculated at the time of admission. The method of use of colistin (IV, IV+inhaler, inhaler), pre-treatment basal laboratory values, focus of infection, isolated microorganisms, duration of intensive care stay, and results were recorded. Serum creatinine levels of the patients were analyzed from the day of colistin initiation. Acute kidney injury staging was performed using RIFLE classification and KDIGO criteria to evaluate renal damage. The patients were divided into two groups, according to the RIFLE classification and KDIGO criteria, with or without acute kidney injury.

\section{Statistical Evaluation}

Statistical analysis of the results obtained in the study was performed using the SPSS (Statistical Package for Social Sciences) 24 program. Descriptive statistics were used for demographic data. The normal distribution suitability of the variables were examined using visual (histogram and probability plots) and analytical methods (KolmogrovSimirnov/Shapiro-Wilk tests). Results are given as numbers and percentages for categorical variables and as mean \pm standard deviations for continuous variables. Independent sample t-test was applied as parametric test to those with normal distribution, and Mann-Whitney $\mathrm{U}$ test as non-parametric test was applied to those who did not show normal distribution. Comparison of the data of the groups with and without AKI was made using Chi-square and Fisher's tests. Kaplan-Meier method was used for survival analysis and compared using log rank analysis. Cox Regression analysis was used for the analysis of variables related to mortality. A p value of less than 0.05 was considered statistically significant.

\section{RESULTS}

The data of 145 patients who were given colistin were analyzed. $86(59.3 \%)$ of these patients were female and $59(40.7 \%)$ were male. The mean age of the patients was $64.9 \pm 16.8$ years. Pneumonia was in the first place with $51 \%$ as the focus of infection. The most common microorganisms isolated in blood culture were $A$. baumanii, K. pneumonia and P. Aeruginosa (50.3\%, $16.6 \%, 10.3 \%$, respectively). Colistin treatment was given as intravenous (IV) to 125 patients, IV and inhaler to 13 patients, and only inhaler to 7 patients.

75 patients were evaluated as AKI when KDIGO criteria were used for the AKI diagnosis, and 54 patients were evaluated as AKI when the RIFLE classification was used. 44 patients were classified as Stage 1, 15 patients as Stage 2, 16 patients as Stage 3 according to KDIGO staging, and 23 patients were classified as Risk, 16 as Injury, 13 as Failure, and 2 as Loss according to RIFLE classification. While the mean age in the group with AKI was calculated as $66.9 \pm 15.5$, the mean age in the group without AKI was calculated as 62.4 \pm 17.9 . Although AKI was more common in elderly patients, no statistical difference was found $(\mathrm{p}=0.183)$. No difference was found between the groups in terms of gender (p: 0.608). AKI did not develop in patients who were given inhaled colistin. No statistically significant difference was found in the development of AKI in those who were given IV or IV+inhaler treatment ( $>0.05$ ). Besides, RRT was applied to 29 of the patients who developed AKI.

The APACHE II scores and SOFA score of the patients were calculated at the time of diagnosis. The mean SOFA score was calculated as $7.91 \pm 2.8$ and the mean APACHE II score as $22.8 \pm 5.3$. In patients who developed AKI, the SOFA score was found as $8.8 \pm 2.9$, and the APACHE II score as $24 \pm 5.4$. In patients who did not develop AKI, the SOFA score was calculated as $6.8 \pm 2.2$, and the APACHE II score as $21.3 \pm 4.7$. SOFA and APACHE II scores were found to be significantly higher in patients with AKI (p: 0.03 , p: 0.027 , respectively). Comparison of the groups by the demographic, clinical and laboratory characteristics is shown in Table 1. 


\begin{tabular}{|c|c|c|c|c|}
\hline & $\begin{array}{c}\text { All Patients (n:145) } \\
\text { Mean } \pm \text { SD }\end{array}$ & Non-AKI (n:70) Mean \pm SD & AKI (n:75) Mean \pm SD & $\mathbf{p}$ \\
\hline $\begin{array}{l}\text { Female } \\
\text { Male }\end{array}$ & $\begin{array}{l}86(59.3 \%) \\
59(40.7 \%)\end{array}$ & $\begin{array}{l}40(57.1 \%) \\
30(42.9 \%)\end{array}$ & $\begin{array}{l}46(61.3 \%) \\
29(38.7 \%)\end{array}$ & 0.608 \\
\hline Age & $64.88 \pm 16.77$ & $62.39 \pm 17.87$ & $66.86 \pm 15.50$ & 0.183 \\
\hline Urea $(\mathrm{mg} / \mathrm{dL})$ & $95.43 \pm 64.71$ & $46.24 \pm 25.88$ & $119.77 \pm 64.38$ & $<0.001$ \\
\hline Creatinine (mg/dL) & $1.92 \pm 1.68$ & $0.62 \pm 0.26$ & $2.56 \pm 1.72$ & $<0.001$ \\
\hline Albumin $(\mathrm{g} / \mathrm{dL})$ & $2.19 \pm 0.42$ & $2.27 \pm 0.49$ & $2.14 \pm 0.38$ & 0.117 \\
\hline Uric acid (mg/dL) & $5.12 \pm 2.84$ & $3.23 \pm 1.61$ & $6.06 \pm 2.86$ & 0.002 \\
\hline SOFA score & $7.9 \pm 2.8$ & $6.8 \pm 2.2$ & $8.8 \pm 2.9$ & 0.03 \\
\hline APACHE II & $22.8 \pm 5.3$ & $21.3 \pm 4.7$ & $24 \pm 5.4$ & 0.027 \\
\hline $\begin{array}{l}\text { Microorganisms } \\
\text { A. baumanii } \\
\text { K. pneumonia } \\
\text { P. Aeruginosa } \\
\text { Another microorganism } \\
\text { No reproduction }\end{array}$ & $\begin{array}{c}73(50.3 \%) \\
24(16.6 \%) \\
15(10.3 \%) \\
17(11.7 \%) \\
16(11 \%)\end{array}$ & $\begin{array}{c}38(54.3 \%) \\
12(17.1 \%) \\
9(12.9 \%) \\
6(8.6 \%) \\
5(7.1 \%)\end{array}$ & $\begin{array}{c}35(46.7 \%) \\
12(16 \%) \\
6(8 \%) \\
11(14.7 \%) \\
11(14.7 \%)\end{array}$ & \\
\hline $\begin{array}{l}\text { Survivor } \\
\text { Ex }\end{array}$ & $\begin{array}{l}80(55.2 \%) \\
65(44.8 \%)\end{array}$ & $\begin{array}{l}44(62.9 \%) \\
26(37.1 \%)\end{array}$ & $\begin{array}{l}36(48 \%) \\
39(52 \%)\end{array}$ & 0.072 \\
\hline ICU length of stay (days) & $22.60 \pm 9.04$ & $24.2 \pm 8.47$ & $21.12 \pm 9.36$ & 0.04 \\
\hline
\end{tabular}

$65(44.8 \%)$ of the patients included in the study died. 52\% (n:39) of patients who developed AKI and 37.1\% (n:26) of those who did not develop AKI died $(\mathrm{p}=0.02)$. Mortality numbers according to AKI classification methods and stages are shown in Table 2.

\begin{tabular}{|c|c|c|c|}
\hline & All patients (n:145) & $\operatorname{Ex}(n: 65)$ & Survivor (n:80) \\
\hline \multicolumn{4}{|l|}{ RIFLE } \\
\hline Non-AKI & $91(62.8 \%)$ & $33(50.8 \%)$ & $58(72.5 \%)$ \\
\hline $\mathrm{R}$ & $23(15.9 \%)$ & $14(21.5 \%)$ & $9(11.3 \%)$ \\
\hline I & $16(11 \%)$ & $8(12.3 \%)$ & $8(10 \%)$ \\
\hline $\mathrm{F}$ & $13(9 \%)$ & $9(13.8 \%)$ & $4(5 \%)$ \\
\hline $\mathrm{L}$ & $2(1.4 \%)$ & $1(1.5 \%)$ & $1(1.3 \%)$ \\
\hline \multicolumn{4}{|l|}{ KDIGO } \\
\hline Non-AKI & $70(48.3 \%)$ & $26(40 \%)$ & 44 (55\%) \\
\hline Stage 1 & $44(30.3 \%)$ & $21(32.3 \%)$ & $23(28.8 \%)$ \\
\hline Stage 2 & $15(10.3 \%)$ & $7(10.8 \%)$ & $8(10 \%)$ \\
\hline Stage 3 & $16(11 \%)$ & $11(16.9 \%)$ & $5(6.3 \%)$ \\
\hline
\end{tabular}

The mean duration of stay in the ICU was $22.60 \pm 9.04$. The duration of stay in the ICU was found to be significantly lower in the group who developed AKI (p:0.04). As per the Kaplan-Meier analysis, 30-day mortality in patients with AKI was significantly higher than both KDIGO staging and RIFLE classification $(\mathrm{p}=0.03, \mathrm{p}=0.005$, respectively) (Figure 1-2)

When assessed by COX regression analysis, the risk of death was 1.42 -fold higher in KDIGO Stage 1 patients, 2.79-fold higher in KDIGO Stage 2 patients, and 2.10-fold higher in KDIGO Stage 3 patients compared to non-AKI patients. When evaluated by the RIFLE classification, the risk of death was 2.32 times higher in the risk group, 3.12 times higher in the Injury group, 2.06 times higher in the Failure group and 2.15 times higher in the Loss group compared to the non-AKI group. (Table 3 )

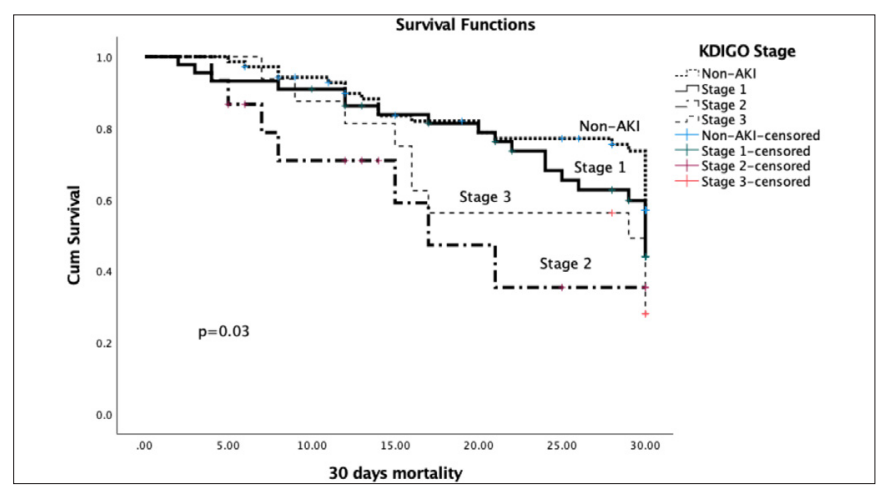

Figure 1. Kaplan-Meier survival analysis for KDIGO Stage

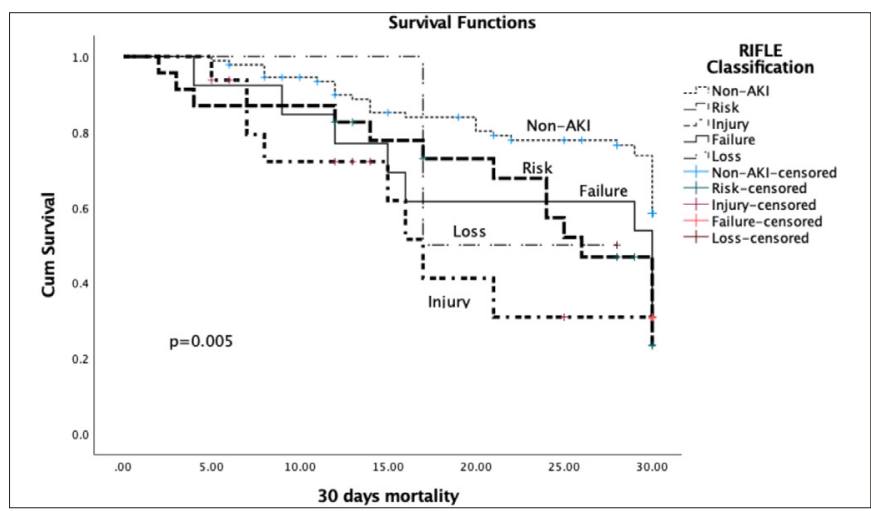

Figure 2. Kaplan-Meier survival analysis for RIFLE Classification

\begin{tabular}{|lccc|}
\hline \multicolumn{4}{|l|}{ Table 3. Cox regression analysis for 30 days mortality } \\
\hline Variables & OR & $\mathbf{9 5 \%}$ CI & p \\
\hline KDIGO & & & \\
Stage 1 & 1.42 & $0.80-2.53$ & 0.226 \\
Stage 2 & 2.79 & $1.19-6.49$ & 0.017 \\
Stage 3 & 2.10 & $1.03-4.25$ & 0.039 \\
RIFLE & & & \\
Risk & 2.32 & $1.23-4.36$ & 0.009 \\
$\quad$ Injury & 3.12 & $1.42-6.84$ & 0.004 \\
$\quad$ Failure & 2.06 & $0.98-4.32$ & 0.053 \\
Loss & 2.15 & $0.29-15-89$ & 0.453 \\
\hline OR: odds ratio, CI: Confidence Interval & & \\
\hline
\end{tabular}




\section{DISCUSSION}

In the present day, colistin has begun to be reused in the treatment of multidrug-resistant gram-negative bacteria due to the limited treatment options available. The most important disadvantage of this drug is that it is nephrotoxic. Nephrotoxicity rates developing due to colistin use vary between $20-60 \%$ (4). This variability in nephrotoxicity rates is thought to arise from different patient populations and the use of various criteria (RIFLE, AKIN, KDIGO) (6).

After the use of colistin, there are studies that have detected AKI using the RIFLE classification. Hartzell et al. (7) reported the incidence of AKI as $45 \%$ in their study including 66 patients. Kwon JA et al. (8) found the incidence of AKI in 71 critical patients as 54\%, and Kaya $\mathrm{M}$ et al. (9) found $69.7 \%$ in patients in the ICU of the oncology hospital. Considering the studies diagnosed with AKI using KDIGO criteria, Günay et al. (4) reported the incidence of AKI as $64.4 \%$ in their study in which they analyzed the data of 149 patients. Giacobbe et al. (10) found the incidence of AKI as $68.8 \%$ in their study including 170 patients, and Yu et al. (11) found $46.7 \%$ in their multi-center study in which 295 patients were included. In our study, we evaluated the development of AKI in patients who were given colistin treatment as per both RIFLE classification and KDIGO criteria. In the study, we found the rate of AKI development as $37.2 \%$ (n:54) when the RIFLE classification was used, and $51.7 \%$ (n:75) when the KDIGO criteria were used. The reason for this difference is that while 1.5-fold increase in serum creatinine level in RIFLE classification is classified as risk group, serum creatinine level $0.3 \mathrm{mg} / \mathrm{dL}$ or baseline creatinine 1.5-2fold increase is accepted as Stage 1 as per the KDIGO criteria. Considering this situation, patients who do not meet the RIFLE classification risk group criteria can be classified as KDIGO Stage 1 AKI. Therefore, the classification preferred for the diagnosis of AKI affects the incidence of AKI. In our study, the number of patients who developed AKI stage 1 was 44 while the number of patients in the RIFLE Risk stage was 23. As a result of our study, we found that KDIGO criteria may be more sensitive in diagnosing AKI.

The risk of developing AKI increases in elderly patients. When the literature is reviewed, AKI after colistin is observed more frequently in elderly patients $(4,12,13)$. In our study, the average age of the patients who developed AKI was higher.

APACHE II and SOFA scores calculated in critically ill patients hospitalized in ICU are used to predict prognosis. Kidney function tests are also evaluated in both scoring systems. The risk of AKI increases in patients with high
APACHE II and SOFA scores (14). In our study, AKI was found to be statistically significantly higher in patients with high APACHE II and SOFA scores calculated at the time of admission to the hospital ( $\mathrm{p}=0.027, \mathrm{p}=0.03$, respectively).

The development of AKI and the advanced stage of AKI increase mortality in patients followed in the ICU (15-17). In our study, we investigated the relationship between AKI and mortality. 52\% of patients who developed AKI and $37.1 \%$ of those who did not develop AKI died $(\mathrm{p}=0.02)$. We compared the mortality rates of our patients with AKI according to KDIGO criteria and RIFLE classification. Mortality was found to be statistically significantly higher in KDIGO Stage 2 and 3 mortality was significantly higher in Risk and Injury group according to RIFLE classification.

Our study has some limitations. The fact that the study has a retrospective design can be considered as the main limitation. Besides, the limited number of our patients due to the fact that the study was single-centered and was not a combined ICU is an important limitation. Multicenter prospective studies involving different patient populations may provide stronger results.

\section{CONCLUSION}

As a result, the use of colistin has become mandatory due to the limited alternative antibiotic treatment options other than colistin in MDR Gram-negative bacterial infections. Our data showed that the incidence of AKI was increased in critically ill patients using colistin. Tthe classification preferred for the diagnosis of AKI affects the incidence of AKI. We think that the KDIGO criteria are more sensitive to diagnose AKI in these patients.

\section{ETHICAL DECLARATIONS}

Ethics Committee Approval: Approval for the study was granted by the Ethics Committee of Dicle University Medical Faculty (Date: 05.03.2020, Decision No: 254).

Informed Consent: Because the study was designed retrospectively, no written informed consent form was obtained from patients.

Conflict of Interest Statement: The authors have no conflicts of interest to declare.

Financial Disclosure: The authors declared that this study has received no financial support.

Author Contributions: All of the authors declare that they have all participated in the design, execution, and analysis of the paper, and that they have approved the final version. 


\section{REFERENCES}

1. Cerceo E, Deitelzweig SB, Sherman BM, et al. Multidrugresistant Gram-negative bacterial infections in the hospital setting: overview, implications for clinical practice, and emerging treatment options. Microb Drug Resist 2016; 22: 412-31.

2. Kınıklı S, Cesur S, Yücel M, Hatipoğlu ÇA, Dinç B. Determination of polymyxin B, minocycline, colistin and phosphomycin susceptibilities in Acinetobacter baumannii strains showing carbapenem resistant multidrug resistance phenotype. J Health Sci Med 2019; 2: 49-53.

3. Falagas ME, Kasiakou SK. Colistin: the revival of polymyxins for the management of multidrug-resistant gram-negative bacterial infections. Clin Infect Dis 2005; 40: 1333-41.

4. Gunay E, Kaya S, Baysal B, Yuksel E, Arac E. Evaluation of prognosis and nephrotoxicity in patients treated with colistin in intensive care unit. Ren Fail 2020; 42: 704-9.

5. Javan AO, Shokouhi S, Sahraei Z. A review on colistin nephrotoxicity. Eur j Clin Pharmacol 2015; 71: 801-10.

6. Inc1 A, Toker MK, Bicer IG, Derbent A, Salihoglu Z. Determination of colistin-related nephrotoxicity and risk factors in intensive care unit. North Clin Istanb 2018; 5: 120-4.

7. Hartzell JD, Neff R, Ake J, et al. Nephrotoxicity associated with intravenous colistin (Colistimethate sodium) Treatment at a Tertiary Care Medical Center. Clinical Infectious Diseases 2009; 48: 1724-8.

8. Kwon JA, Lee JE, Huh W, et al. Predictors of acute kidney injury associated with intravenous colistin treatment. Int J Antimicrob Agents 2010; 35: 473-7.

9. Kaya M, Tunçel Yİ, Kuru RN, et al. Onkoloji hastanesi yoğun bakım ünitesinde kolistin ilişkili nefrotoksisitenin retrospektif değerlendirilmesi. Türk Yoğun Bakım Derneği Derg 2014; 12: 51 6.

10. Giacobbe DR, Masi AD, Leboffe L, et al. Hypoalbuminemia as a predictor of acute kidney injury during Colistin treatment. Sci Rep 2018 10; 8: 11968.

11. Yu SN, Kim T, Park SY, et al. Predictors of acute kidney injury and 28-day mortality in carbapenem-resistant Acinetobacter baumannii complex bacteremia. Microb Drug Resist 2021; 10.1089/mdr.2020.0312.

12. Ciftci A, Izdes S, Altintaş ND. Factors determining nephrotoxicity and mortality in critical care patients receiving colistin. J Infect Dev Ctries 2018; 11: 912-8.

13. Deryke CA, Crawford AJ, Uddin N, Wallace MR. Colistin dosing and nephrotoxicity in a large community teaching hospital. Antimicrob Agents Chemother 2010; 54: 4503-5.

14. Søvik S, Isachsen MS, Nordhuus KM, et al. Acute kidney injury in trauma patients admitted to the ICU: a systematic review and meta-analysis. Intensive Care Med 2019; 45: 407-19.

15. Aydin E, Kadiroglu AK, Yilmaz Aydın F, et al. The relationship between acute kidney injury and inflammation-based parameters and mortality in oncologic intensive care patients. J Crit Intensive Care 2020; 11: 50-4.

16. Vieira JM Jr, Castro I, Curvello-Neto A, et al. Effect of acute kidney injury on weaning from mechanical ventilation in critically ill patients. Crit Care Med 2007; 35: 184-91.

17. Hoste EAJ, Bagshaw SM, Bellomo R, et al. Epidemiology of acute kidney injury in critically ill patients: the multinational AKI-EPI study. Intensive Care Med 2015; 41: 1411-23. 